\title{
Rapprochement mécaniciens-physiciens
}

Ces dernières années ont vu un intérêt grandissant des physiciens pour des problèmes de mécanique : hydrodynamique, turbulence, fracture, tribologie, milieux granulaires... En effet, ces domaines recèlent de beaux problèmes d'instabilités, d'effets non linéaires ou de singularités. Les jeunes générations de physiciens et de mécaniciens ont convergé sur la nécessité de descriptions multi-échelles et de systèmes modèles. Si l'interaction avec les mécaniciens autour de la mécanique des fluides a été un beau succès, le dialogue est beaucoup moins avancé dans le domaine de la mécanique des solides.

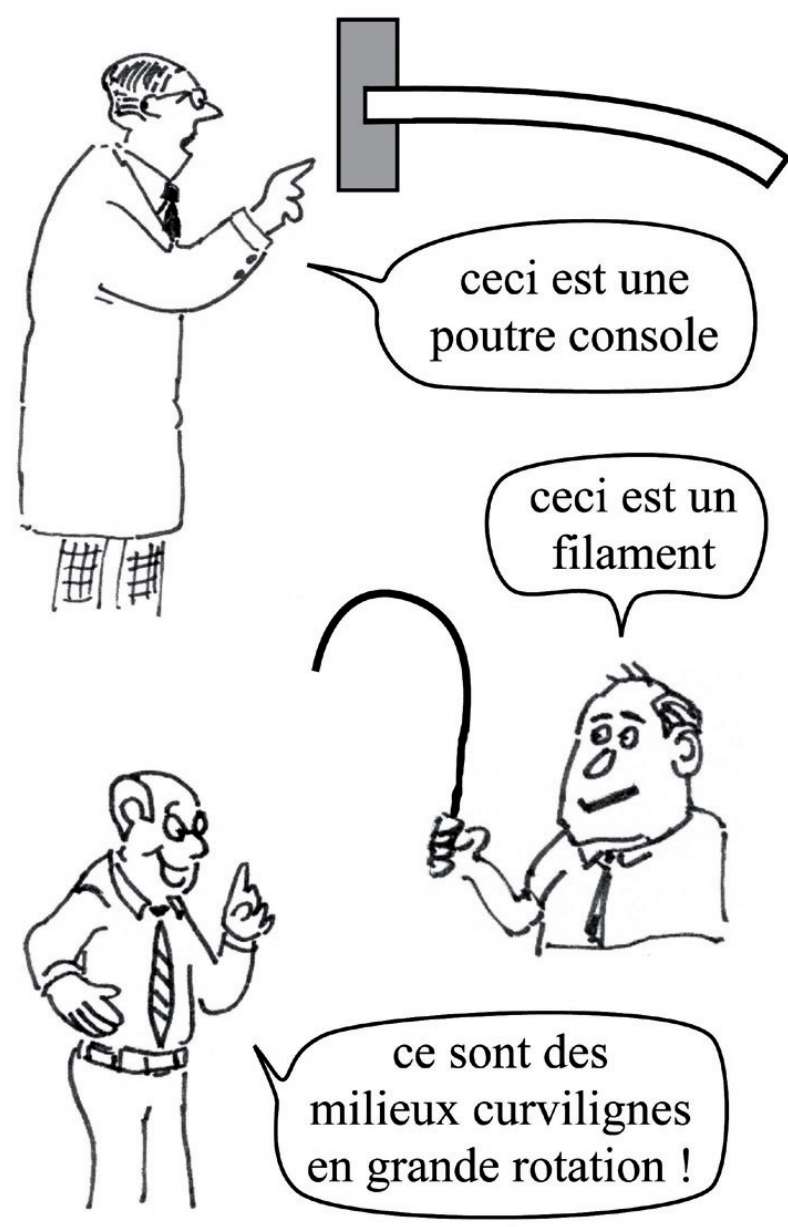

Les figures de cet article sont dues à Basile Audoly, José Bisco, Nicolas Clauvelin, Sébastien Neukirch et Benoît Roman.

(1) L'AUM est un groupe thématique transverse de l'Association Française de Mécanique (www.afm.asso.fr).
Jeudi 2 avril 2009, une première rencontre entre mécaniciens et physiciens a été organisée conjointement par l'AUM (Activités Universitaires en Mécanique $\left.{ }^{(1)}\right)$ et la SFP à l'Institut Henri Poincaré, autour de questions actuelles sur les tiges élastiques. Elle a rassemblé 47 participants autour d'un ensemble d'exposés provenant des deux communautés, avec le réel désir de dépasser les différences de langages ou de méthodes. Cela a fourni l'occasion de confronter les points de vue et les questions des deux communautés sur un sujet précis.

Du point de vue des mécaniciens, les questions qui se posent étaient de deux ordres.

Peut-on décrire un objet élancé (dont la section est petite par rapport à la longueur) comme une simple "ligne élastique »? Comment en choisir l'«élasticité "?

Si l'on souhaite décrire une poutre par une ligne, se pose le problème de la description géométrique, qui peut être très complexe (entortillement, auto-contact...). Dans ce cas, on ne peut se contenter de repérer la géométrie de la ligne moyenne : une approche possible (Alexandre Watzky) consiste à utiliser un repère dit "matériel », qui est attaché à la section transverse de la poutre. Une autre approche consiste à appliquer la théorie de l'élasticité à la tige, dont on fait ensuite tendre l'épaisseur asymptotiquement vers zéro. Une classification de modèles de poutres, en fonction de l'ordre de grandeur des forces appliquées, est proposée (Jean-Jacques Marigo) : une même poutre peut être vue comme une tige élastique ou comme un fil (infiniment flexible) selon l'ordre de grandeur des forces qu'on lui applique. Cette démarche est reprise (Olivier Millet) pour des tiges à section très aplatie " poutres-voile " (comme celles d'un mètre à enrouleur) et montre ainsi que le modèle usuel des ingénieurs (dû à Vlassov) n'est pas correct, car il néglige des couplages entre la torsion et la flexion. Une étude expérimentale a été présentée et montre clairement que cet effet de couplage existe, comme le prédit le modèle asymptotique construit par l'auteur.

Comment simuler ces structures dont le comportement non linéaire donne lieu à des instabilités?

Une méthode asymptotique numérique (Michel Potier-Ferry) permet le suivi des branches de solutions d'équations aux dérivées partielles non linéaires et la détection des bifurcations. Le principe de la méthode consiste à rechercher les branches de solutions, sous la forme d'un développement perturbatif dont les caractéristiques donnent les points de bifurcations. Cette méthode est appliquée à la recherche d'instabilités de claquage ou de flambage des plaques et coques élastiques. Dans certains procédés de mise en forme, tel que le laminage, l'apparition des contraintes résiduelles est inévitable et peut engendrer une instabilité de flambage. 


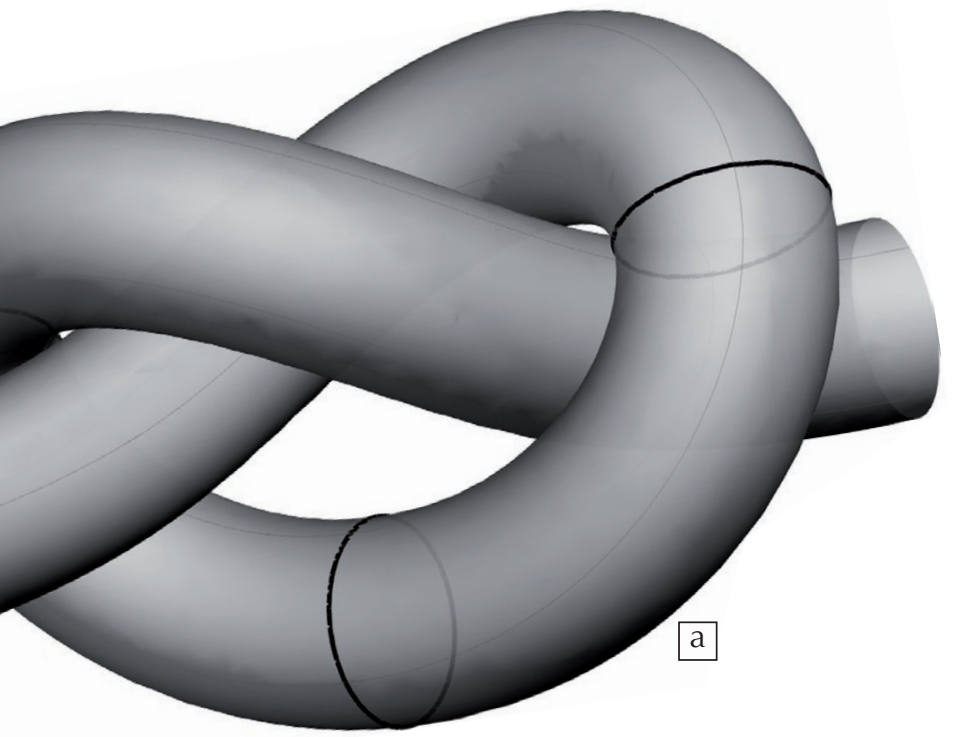

Les questions posées par les physiciens sur des tiges élastiques pouvaient se classer en trois types.

L'état d'une feuille de papier froissée et mise en boule ne peut être décrit que de manière statistique, et ces systèmes ne subissent pas d'agitation thermique... Pourtant le traitement statistique (Arezki Boudaoud) des nombreux états d'équilibre d'une tige très confinée montre que les distributions d'énergie de deux types de plis en présence sont identiques, suggérant une thermalisation... Ce sont des questions qu'on retrouve, par exemple, dans les milieux granulaires.

Ensuite, l'effet, sur les poutres élastiques, de forçages peu habituels en mécanique, prend une nouvelle importance avec la miniaturisation. Ainsi, la tension de surface (Sébastien Neukirch) peut faire flamber une structure mince qui sort d'un bain de liquide mouillant (fig. 1). L'émission d'électrons par un nanotube chargé (Sorin Perisanu) peut conduire à une instabilité oscillante, dont l'étude requiert des outils de mécanique et d'électrostatique.

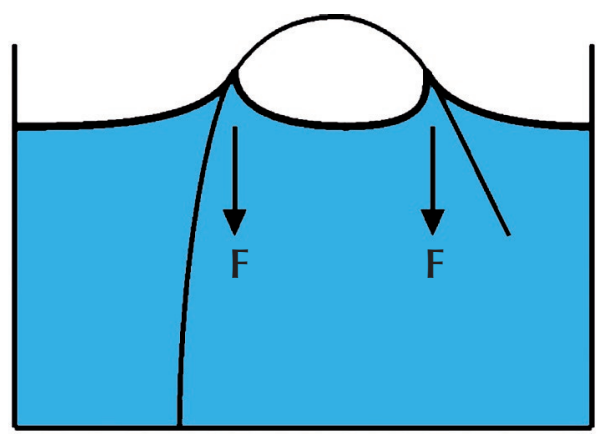

1. Une lamelle élastique peut être fortement courbée par des forces capillaires. L'équilibre dépend de la rigidité de la lamelle, de la tension de surface, et de la hauteur de bain. La non-linéarité du problème fait que de multiples configurations d'équilibre stables coexistent pour la même hauteur de bain.

Enfin, les techniques de couches limites et de raccordement permettent de résoudre des problèmes qui font intervenir des points singuliers à la frontière entre des zones où des approximations différentes s'appliquent. On montre ainsi (S. Neukirch) que l'autocontact d'une tige qui forme un nœud est ponctuel si l'épaisseur est nulle, mais s'étend sur une zone continue et des

2. Mécanique des nœuds élastiques. (a) Noeud de trèfle serré. On tire sur les extrémités d'un filament noué : quelle est la configuration du système lorsque la tension devient (très) grande? (b) Noud de trèfle en équilibre élastique sous une tension appliquée T. On distingue trois régions : la boucle, la tresse et les parties terminales. Le contact se produit dans la région de tresse où les deux brins se touchent le long d'un arc de cercle de rayon de courbure double de celui de la boucle.

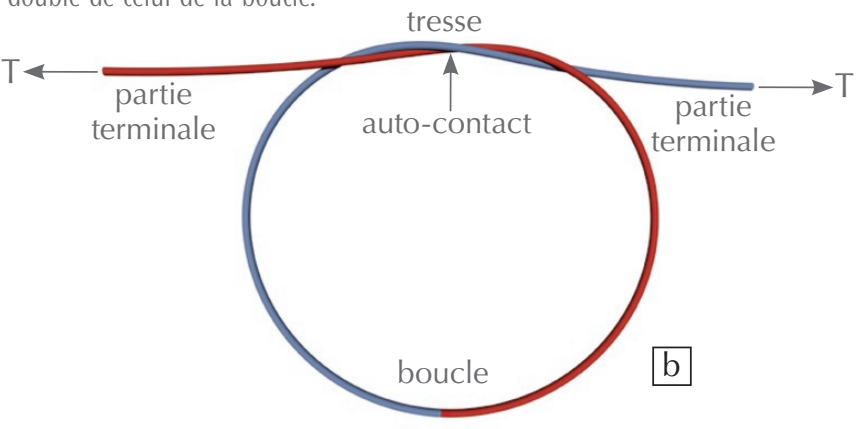

points discrets dès que l'épaisseur est finie (fig. 2). D'autre part, les modes propres fondamentaux de coques de courbure gaussienne positive ou négative sont de nature différente de ceux d'une plaque de courbure nulle, où il existe un mode isométrique : Yves Pomeau utilise pour démontrer ces résultats des techniques de couches limites pour estimer les modes de vibration d'un tore qui contient deux lignes où la courbure gaussienne est nulle, séparant deux zones de courbures opposées.

Un programme interdisciplinaire pour le collège (Étienne Guyon) a été illustré par l'étude des fibres (du muscle au tissu), qui fait appel à une approche mécanique absente dans les formations. Deux caractéristiques communes des fibres naturelles se dégagent : leur construction hiérarchique et l'importance des effets entre fibres élémentaires courtes dans le comportement mécanique.

Il était particulièrement intéressant que É. Guyon et Y. Pomeau participent à cette journée, puisqu'ils ont tous deux joué un rôle très important dans l'intérêt croissant que les physiciens portent à la mécanique : É. Guyon en créant et animant un laboratoire de physique et mécanique sous l'impulsion de P.G. de Gennes, et Y. Pomeau en mettant en lumière les beaux problèmes de singularités qui peuvent apparaitre quand on froisse une plaque mince.

Les fichiers utilisés par les orateurs comme supports de leurs présentations sont disponibles à l'adresse suivante : www.pmmh.espci.fr/ benoit/AUMSFP

En fin de journée, une table ronde réunissant les participants et les organisateurs a permis aux questions suivantes d'être discutées : mise en place d'autres rencontres courtes analogues sur d'autres sujets à l'intersection mécanique-physique (instabilités, rupture, etc.), école d'été en mécanique des solides, création d'une division commune AUM-SFP, invitations croisées de membres des deux sociétés aux commissions pédagogiques.

Étienne Guyon ${ }^{1}$, Sébastien Neukirch ${ }^{2}$ et Benoît Roman' ${ }^{1}$ (benoit@pmmh.espci.fr)

1 - Physique et mécanique des milieux hétérogènes, ESPCI, 10 rue Vauquelin, 75231 Paris Cedex 05 2 - Institut Jean le Rond d'Alembert,

CNRS et Université P. et M. Curie, 4 place Jussieu, 75005 Paris 